\title{
Shorter Method for Computing the Coefficient of Correlation
}

\section{Leonard P. Ayres}

To cite this article: Leonard P. Ayres (1920) Shorter Method for Computing the Coefficient of Correlation, The Journal of Educational Research, 1:3, 216-221, DOI: 10.1080/00220671.1920.10879044

To link to this article: http://dx.doi.org/10.1080/00220671.1920.10879044

册 Published online: 15 Dec 2014.

Submit your article to this journal $[\pi$

Q View related articles $₫$ 


\title{
SHORTER METHOD FOR COMPUTING THE COEFFICIENT OF CORRELATION
}

\author{
LEONARD P. AYRES \\ Russell Sage Foundation, New York City
}

The coefficient of correlation measures the agreement between two series of numbers. The numbers are in pairs and each number in the first series is the first member of a pair of measurements, while the corresponding number in the second series is the second member of that pair. The object of computing the coefficient of correlation is to find in what degree smaller quantities in one series imply correspondingly smaller quantities in the other, and larger quantities in the one imply correspondingly larger quantities in the other. The coefficient of correlation does this by comparing the amount of agreement that does exist with the perfect agreement that might exist.

\section{The Pearson Coefficient}

The formula for finding the Pearson coefficient of correlation is best expressed as:

$$
r=\frac{\Sigma(x \cdot y)}{\sqrt{\Sigma x^{2}} \sqrt{\Sigma y^{2}}}
$$

In this formula:

$$
\begin{aligned}
& r=\text { correlation } \\
& \Sigma=\text { summation } \\
& x=\text { deviations from the average of the first series } \\
& y=\text { deviations from the average of the second series }
\end{aligned}
$$

The use of the formula may be illustrated by finding the correiation between two short and simple series as follows:

\begin{tabular}{rr}
3 & 6 \\
4 & 5 \\
5 & 7 \\
8 & 11 \\
10 & 11 \\
\hline 30 & 40
\end{tabular}

Since there are five numbers in each series, the average of the first series is 6 , and that of the second is 8 . The next step, according 
to the formula, is the finding of the deviations of these numbers away from their averages. Calling these deviations $x$ and $y$ they are as follows:

$\begin{array}{cc}x & y \\ -3 & -2 \\ -2 & -3 \\ -1 & -1 \\ +2 & +3 \\ +4 & +3\end{array}$

It is clear that there is considerable agreement between the general trends of these two series. Large minus deviations in the first series are accompanied by large minus deviations in the second series and large plus deviations in the first by large plus deviations in the second. The problem is to find how nearly complete this agreement is; how near the partial agreement that does exist comes to equaling the perfect agreement that might exist.

\section{The Actual Deviations}

This involves comparing the actual deviations of these two series with those that might be found if the agreement were perfect. The actual deviations are measured by multiplying the corresponding members of the two series and getting the sum of these products as follows:

$$
\begin{aligned}
& x y \\
& +6 \\
& +6 \\
& +1 \\
& +6 \\
& +12 \\
& \hline 31
\end{aligned}
$$

\section{Deviations for Perfect Agreement}

The next step is to find a measure of the deviations that would exist if the agreement between the two series were perfect. The first deviation of the $x$ series is -3 and the second is -2 . If the corresponding deviations of the second series were identical they would also be -3 and -2 and there would be perfect agreement.

Following the method already used in measuring the actual deviations, a measurement may be found for the deviations that would exist if all those of the second series were equal to those 
in the first. This is done by multiplying all these deviations of the first series by themselves or finding their squares. The same process is now carried through with the second series and the squares of both series of deviations are the following:

\begin{tabular}{rr}
$x^{2}$ & $y^{2}$ \\
9 & 4 \\
4 & 9 \\
1 & 1 \\
4 & 9 \\
16 & 9 \\
\hline 34 & -32
\end{tabular}

We now have three quantities: 31 which measures the actual deviations of the two series, 34 which shows what the deviations of the $x$ series would amount to if they were accompanied by identical deviations in the $y$ series, and 32 which shows what those of the $y$ series would amount to if they were accompanied by identical deviations in the $x$ series.

Comparing the Actual with the Possible

The actual deviations must now be compared with those that might exist if the correlation were perfect. This is done by comparing 31 with 34 and 32 simultaneously and, since 34 and 32 are the sums of series of squares, the comparison is made by finding the product of their square roots and comparing 31 with that number. This working out of the formula gives the following:

$$
r=\frac{\Sigma x . y}{\sqrt{\Sigma x^{2}}+\overline{\Sigma y^{2}}}=\frac{31}{\sqrt{34} \sqrt{32}}=\frac{31}{32.98}=0.94
$$

The work described is easy in the artificially simple example chosen but far more laborious in cases where the average is not a whole number, for this involves multiplying and squaring with numbers having decimal fractions. There are methods for doing this that involve finding the deviations from a whole number near to the average and then correcting the work to allow for the differences between the results so found and the correct results. These methods are laborious and increase the chances of letting errors of computation destroy the accuracy of the work.

\section{A Shorter Method}

All these difficulties may be avoided by a method which it is the object of this article to present, and which gives the sums of 
the products and the sums of the squares of the deviations directly from the squares of the original numbers. It does not involve arranging the series in order, or finding the separate deviations, and so avoids the necessity for taking into account the plus and minus signs of deviations.

This method is based on considering every number in a series as being equal to the average of the series plus a plus or minus deviation from that average. Thus 10 , which is the last number in the first series of the illustrative example already cited, may be considered as being equal to the average of the series, or 6 , plus the deviation 4.

If now we find the sum of the squares of the numbers in a series and subtract from it the product found by multiplying the square of the average of the series by the number of cases, the remainder will be the sum of the squares of the deviations from the average. Suppose, for example, that the series consists of the two numbers 4 and 8 . Their average is 6 and the deviations from it are -2 and +2 , the sum of the squares of which equals 8 . But it is also true that the squares of these numbers are 16 and 64 with a sum of 80 . If we subtract from this the square of the average, or 36 . multiplied by the number of cases, or 2, we again get 8 as a result.

\section{The Formula}

Utilizing this principle we may compute coefficients of correlation directly from the products and squares of the items of the two series without finding the separate deviations. Before writing the formula certain abbreviations may be set down as follows:

$$
\begin{aligned}
& \text { Sub. }=\text { the first, or subject series } \\
& \text { Rel. }=\text { the second, or relative series } \\
& \text { Tot. }=\text { the total of a series, the sum of its items } \\
& \text { Av. }=\text { the average of a series }
\end{aligned}
$$

The formula expressed in these abbreviations, and also using the letters $S$ and $R$ for subject and relative, is as follows:

Sum of products of subject and relative items minus

av. of subject items $X$ tot. of relative items

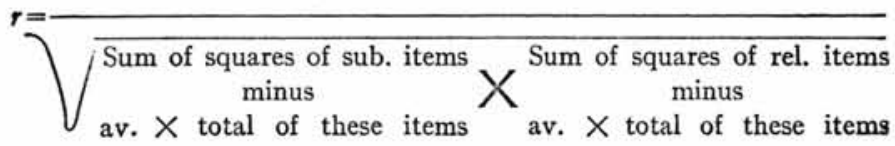


It may be more easily written but perhaps less easily remembered if expressed as follows:

$r=\frac{\Sigma S . R-\frac{\Sigma S . \Sigma R}{N}}{\sqrt{\left[\Sigma S^{2}-\frac{(\Sigma S)^{2}}{N}\right]\left[\Sigma R^{2}-\frac{(\Sigma R)^{2}}{N}\right]}}$

The application of this formula to the example already worked by the conventional method is as follows:

\begin{tabular}{|c|c|c|c|c|c|}
\hline & Sub. & Rel. & Sub. ${ }^{2}$ & Rel. $^{2}$ & Sub. $\times$ \\
\hline & 3 & 6 & 9 & 36 & 18 \\
\hline & 4 & 5 & 16 & 25 & 20 \\
\hline & 5 & 7 & 25 & 49 & 35 \\
\hline & 8 & 11 & 64 & 121 & 88 \\
\hline & 10 & 11 & 100 & 121 & 110 \\
\hline & - & - & 一 & - & - \\
\hline Total & 30 & 40 & 214 & 352 & 271 \\
\hline Average & 6 & 8 & & & \\
\hline First corr & rection & $1-(6)$ & $=271-$ & $=31$ & \\
\hline Second co & orrectic & $214-$ & $0)=21$ & $80=34$ & \\
\hline Third cor & rrectior & $52-(8$ & $=352$. & $0=32$ & \\
\hline & & 31 & 31 & & \\
\hline & & 83 & .98 & & \\
\hline
\end{tabular}

An important feature of this method is that $t$ facilitates the use of mechanical aids to computation. The work consists almost entirely of taking squares from tables and multiplying and adding, which are the operations most easily done by machines. It eliminates the long series of subtractions which are not readily done by the help of machines. It also eliminates the necessity for watching for unlike signs in the multiplication of deviations, and making the series of corrections to allow for the differences between the guessed averages and the true averages of the series.

Experience in using this formula in dealing with the problems arising in the regular work of a statistical office indicates that it reduces by something more than one-half the time necessary to compute correlations from short series of 20 or 30 pairs of items. On longer series the saving is greater and on distribution tables involving some thousands of cases, grouped in about 100 compart- 
ments, it cuts the time to one-third or one-fourth of that required by the old methods. Almost more important is the fact that its use very greatly reduces the number of errors made in working out the computations.

The use of the theorem that the sum of the squares of the deviations of a series of items away from their average is equal to the sum of the squares of the items minus the product of the total of the series and its average will greatly facilitate the finding of the standard deviation. Those who are interested in seeking a further explanation of the theorem itself will find it in considering each item of the series as a binomial consisting of the average plus a plus or minus deviation from the average. By squaring a series of such binomials and adding them, it will be found that the middie terms cancel out, leaving the squares of the average and the squares of the deviations. A similar procedure in multiplying the items of the two paired series will explain the process followed in deriving the numerator of the fraction given in the new formula. 